\title{
Effect of Cognitive Behavioral Intervention Therapy on Immune Index in Patients with Breast Cancer
}

\author{
Ping Huang1, Juncheng Guo ${ }^{2,3}$, Min Guo ${ }^{4}$, Yijun Yang², Xiangling Jiang,4, Weidong Cen ${ }^{5}$ \\ ${ }^{1}$ University of South China, Hengyang, China \\ ${ }^{2}$ Xiangya School of Medical Affiliated Haikou Hospital, Haikou, China \\ ${ }^{3}$ Central South University, Changsha, China \\ ${ }^{4}$ Department of Psychology, Hainan General Hospital, Haikou, China \\ ${ }^{5}$ Lin Gao County People's Hospital of Hainan Province, Hainan, China \\ Email: ^g2002m@163.com
}

How to cite this paper: Huang, P., Guo, J.C., Guo, M., Yang, Y.J., Jiang, X.L. and Cen, W.D. (2019) Effect of Cognitive Behavioral Intervention Therapy on Immune Index in Patients with Breast Cancer. $A d$ vances in Breast Cancer Research, 8, 77-85. https://doi.org/10.4236/abcr.2019.82006

Received: May 5, 2019

Accepted: April 27, 2019

Published: April 30, 2019

Copyright $\odot 2019$ by author(s) and Scientific Research Publishing Inc. This work is licensed under the Creative Commons Attribution International License (CC BY 4.0).

http://creativecommons.org/licenses/by/4.0/

\begin{abstract}
Objective: To explore the effect of cognitive behavioral intervention therapy on the cellular immune function of patients undergoing radical mastectomy for the first time, and to provide a scientific basis for improving the treatment of breast cancer patients. Methods: 100 patients with breast cancer who were eligible for admission to the Department of Breast Surgery, Hainan Provincial People's Hospital from January 2016 to December 2017 were selected as subjects. It is the first time to improve the radical mastectomy. They were randomly divided into control group and intervention group, 50 cases each. The control group used conventional treatment and routine care. On the basis of this, the intervention group conducted cognitive behavioral interventions including cognitive reconstruction and behavioral therapy. The patients were measured for serum IL-2, IL-4, IL-6, IL-8 and TNF- $\alpha$ levels on the second day after admission and three months after discharge. The changes of serum IL-2, IL-4, IL-6, IL-8 and TNF- $\alpha$ levels in the two groups were compared before and after cognitive intervention. Results: The concentrations of IL-2, IL-4, IL-6, IL-8 and TNF- $\alpha$ in the serum of the intervention group and the control group before intervention were compared. The difference was not statistically significant $(\mathrm{P}>0.05)$. After the intervention, the serum levels of IL-2, IL-4 and IL- 8 in the intervention group were lower than before the intervention. The concentration of TNF- $\alpha$ was higher than before the intervention. The difference was statistically significant $(\mathrm{P}<0.05)$. Conclusion: Cognitive behavioral intervention therapy can change the levels of IL-2, IL-4, IL-6, IL-8 and TNF- $\alpha$ in serum of breast cancer patients. It has a positive effect on the regulation of immune function in breast cancer patients.
\end{abstract}




\section{Keywords}

Cognitive Behavioral Therapy, Breast Cancer, Stress Response, Coping Style

\section{Introduction}

In recent years, with the improvement of people's living environment, lifestyle and life pressure, the incidence and mortality of cancer have shown an upward trend. Breast cancer is one of the most common tumors in women worldwide, and its incidence is the first in female malignancies [1]. In the course of treatment, breast cancer patients must bear the pain of the disease itself and the side effects caused by the treatment, as well as the psychological pain caused by the loss of the breast due to the loss of the breast. These physical and psychological factors will lead to serious psychological stress such as anxiety, depression, fear and other negative emotions. These negative emotions affect the patient's therapeutic efficacy, resulting in low immune function, which in turn affects the recovery and outcome of the disease. Cognitive behavioral therapy is a group of methods that change thinking or beliefs and behaviors. That is, through an intervention that combines cognitive correction with behavioral correction, based on the patient's existing perceptions, giving cognitive intervention to patients, correcting patients' understanding of disease errors, and correcting their bad behaviors is an intervention combined behavior with cognition. The role of cytokines in the body is primarily to mediate immune and inflammatory responses. A number of studies have shown that the psychosocial factors of stress affect a number of cytokines in the body [2]. This article provides cognitive behavioral intervention therapy for patients who have undergone the first radical mastectomy reform to investigate its effects on interleukin-2 (IL-2), interleukin-4 (IL-4), interleukin-6 (IL-6), interleukin-8 (IL-8) in breast cancer patients. The effect of tumor necrosis factor- $\alpha$ (TNF- $\alpha$ ) levels, is reported as follows. The immunological indicators of breast cancer patients, serum IL-2, IL-4, IL-6, IL-8 and TNF- $\alpha$ are the most sensitive and also the most scientific.

\section{Data and Methods}

\subsection{General Information}

100 patients who were diagnosed as breast malignant tumors in the Department of Breast Surgery of Hainan Provincial People's Hospital from January 2016 to December 2017 and met the inclusion criteria were selected. All women are the first patients to undergo radical mastectomy. Inclusion criteria: 1 pathological, B-ultrasound diagnosis of early and mid-stage breast cancer patients; Psychological factors cancer patients are subject to the same psychological stress and trauma. Most patients have no medical knowledge, and they are unable to identify the stage of tumor development. As long as they have tumors, they are considered to be extremely unfortunate. Psychological intervention and cognitive 
reconstruction have similar academic significance to patients. Therefore, cognitive reconstruction and behavioral therapy for breast cancer patients are not staged. The diagnosis of breast cancer is currently the only method used internationally and domestically.

Patients are older than 18 years of age without other serious systemic disease history or mental history; 4 have basic literacy skills and are able to complete the questionnaire independently, and participate in the study voluntarily.

Exclusion criteria: 1 advanced breast cancer patients; 2 patients older than 65 years of age; 3 suffering from mental illness; 4 with serious heart, liver, kidney and other dysfunction or other history of malignant tumors; 5 do not have basic literacy skills, cannot complete the questionnaire independently or refuse to participate in the study. Randomly divided into intervention group and control group, the intervention group performed cognitive behavioral interventions on routine treatment regimens and the control group received routine treatment. The average age of the intervention group and the control group were (53.16 \pm 9.34$)$ years old and $(54.16 \pm 8.69)$ years old; The body weight was $(62.54 \pm 10.12) \mathrm{kg}$ and $(64.11 \pm$ 12.03) kg; The educational level is $14 / 19 / 27$ and $12 / 20 / 18$ (junior high school and below, high school (secondary school), junior college and above); There was no significant difference in age and weight between the two groups $(P>0.05)$. The educational level, occupation, medical payment method, ethnic and other demographic data were compared between the two groups, There was no statistical significance $(\mathrm{P}>0.05)$. There is comparability between the two groups.

\subsection{Method}

\subsubsection{Intervention Method}

Tumor patients were randomly assigned to the intervention group and the control group after admission. Then a visit was conducted to explain the purpose of the study, to gain their trust, agree to participate in this study, complete the general information questionnaire, master the general situation of patients, and establish a database. The psychological scale of the two groups of patients was assessed on the day of admission and the psychological characteristics were mastered. The control group received routine treatment plus nursing, including reminding patients of nutritional diet, paying attention to the presence or absence of exudate and swelling in the venipuncture area, timely detection of possible adverse reactions and complications of the patient, and carry out routine care such as targeted treatment; The intervention group used the corresponding cognitive behavior intervention based on the psychological stress status on the basis of routine treatment. The intervention time was on the day of admission, 1 day before surgery, 3 days after surgery, and 1 day before discharge. Each patient was psychologically intervened for 30 - 40 min. Intervention methods are mainly cognitive reconstruction and behavioral therapy.

1) Cognitive Reconstruction

According to the patient's psychological condition, take corresponding measures to help patients understand breast cancer correctly, dispel the pa- 
tient's unreasonable cognition and let the patient know that a good psychological state can promote the recovery of the disease and help the patient to build confidence in the disease. It mainly includes correcting the patient's misconceptions, organizing patients and their families to participate in breast cancer treatment common sense missions, detailed description of radical mastectomy, possible adverse reactions during treatment, and promptly answering any questions of each patient and their families.

2) Behavioral Therapy

It mainly adopts progressive muscle relaxation training at home and abroad, progressive muscle relaxation training, abbreviation PMRT, a type of relaxation therapy. This method is a training method that has a certain order and gradually relaxes the muscles. PMRT mainly involves 16 groups of muscles [3]. The method of operation is to record the training process by the instructor and play it out in the form of $\mathrm{mp} 3$. In a relatively quiet and comfortable environment, Allow the patient to empty the stool before training, then let the patient rest for a while, about 10 minutes, and then let the patient train as specified. The progressive muscle relaxation training method is: the trainer starts with the hand in the instruction, followed by the upper limb, shoulder, head, neck, chest, abdomen, lower limbs, and finally the lower limbs. Generally, it is left and right, or right and left. If the trainer first relaxes the left arm and then the right arm, the remaining action can follow this principle. In the course of practice, during the process of guiding the patient's muscle tension, the action is done for $10-15$ seconds, and during the relaxation process, it takes a little longer, that is, 15 - 20 seconds; The training was repeated 3 times for each group of muscles in order; the training time was generally selected at noon and before going to sleep, each for 30 minutes. After the whole body muscles relax, the trainer then carries out imagery imagination, such as imagining beautiful things, pleasing scenes or image pictures. You can also imagine cancer cells as soft persimmons or other things that are broken. It can be crushed by its own powerful immune cells, and finally discharged outside the body like garbage. The time to implement the method should be arranged as close as possible to half an hour before bedtime, from the first day of the intervention, and the time to practice is $15-25$ minutes per day, avoiding interruptions or interruptions throughout the exercise.

\subsubsection{Determination of Immune Indicators}

The determination of each immune index includes tumor necrosis factor IL-2, IL-4, IL-6, IL-8 and cytokine TNF- $\alpha$. The patients in the two groups were tested for the concentration of cellular immune factors on the second day after admission and three months after discharge. All patients were collected from the fasting venous blood at 6:00-7:00 pm on the second day after admission. $3 \mathrm{ml}$ serum samples were collected from the patients, injected into the separation gel, and recorded in the frozen blood plasma. When used, the sample was thawed and centrifuged (1000 g, $15 \mathrm{~min}$ ), and tested after dispensing. The remaining samples were stored at $-80^{\circ} \mathrm{C}$. After the sample was collected, the concentration of each 
immunocytokine in the serum was determined by the professional according to the enzyme-linked immunosorbent assay (ELISA) method.

\subsection{Statistical Methods}

Statistical analysis was performed using SPSS22.0 statistical software. Measurement data were used $x \pm \mathrm{s}$ for description. The paired sample t-test was used to analyze immunological indicators of the two groups; the count data were described by the percentage of cases. The comparison between the two adopted $x^{2}$ test. The difference was statistically significant at $\mathrm{P}<0.05$.

\section{Results}

\section{Comparison of Immunological Indicators before and after Intervention in Breast Cancer Patients}

The results showed that the concentrations of IL-2, IL-4, IL-6, IL-8 and TNF- $\alpha$ in the serum of the intervention group and the control group before intervention were compared. The difference was not statistically significant $(\mathrm{P}>0.05)$. After the intervention, the IL-2, IL-4 and IL-8 levels in the intervention group were lower than those before the intervention, and the TNF- $\alpha$ concentration was higher than that before the intervention $(\mathrm{P}<0.05)$. See Table 1 for details.

Table 1. Comparison of immunological indicators before and after intervention in two groups of patients $(\bar{x} \pm s, \mathrm{pg} / \mathrm{ml})$.

\begin{tabular}{|c|c|c|c|c|c|}
\hline \multicolumn{2}{|c|}{ Immune index } & $\begin{array}{l}\text { Intervention Group } \\
\qquad(\mathrm{n}=50)\end{array}$ & $\begin{array}{l}\text { Comparison Group } \\
(\mathrm{n}=50)\end{array}$ & $t$ & $P$ \\
\hline \multirow{2}{*}{ IL-2 } & $\begin{array}{c}\text { Before } \\
\text { intervention }\end{array}$ & $12.49 \pm 3.09$ & $12.56 \pm 5.12$ & 0.083 & 0.467 \\
\hline & $\begin{array}{c}\text { After } \\
\text { intervention }\end{array}$ & $9.19 \pm 6.39$ & $11.34 \pm 5.35$ & 1.824 & 0.036 \\
\hline \multirow{2}{*}{ TNF- $\alpha$} & $\begin{array}{c}\text { Before } \\
\text { intervention }\end{array}$ & $6.24 \pm 3.64$ & $6.38 \pm 3.46$ & 0.197 & 0.422 \\
\hline & $\begin{array}{c}\text { After } \\
\text { intervention }\end{array}$ & $21.27 \pm 5.38$ & $8.39 \pm 4.75$ & 12.690 & 0.000 \\
\hline \multirow{2}{*}{ IL-4 } & $\begin{array}{c}\text { Before } \\
\text { intervention }\end{array}$ & $8.25 \pm 4.24$ & $8.01 \pm 5.34$ & 0.249 & 0.402 \\
\hline & $\begin{array}{c}\text { After } \\
\text { intervention }\end{array}$ & $5.35 \pm 3.98$ & $7.98 \pm 3.76$ & 3.396 & 0.000 \\
\hline \multirow{2}{*}{ IL-6 } & $\begin{array}{c}\text { Before } \\
\text { intervention }\end{array}$ & $15.24 \pm 4.87$ & $15.34 \pm 4.24$ & 0.109 & 0.456 \\
\hline & $\begin{array}{c}\text { After } \\
\text { intervention }\end{array}$ & $14.93 \pm 5.46$ & $16.13 \pm 4.34$ & 1.217 & 0.113 \\
\hline \multirow{2}{*}{ IL-8 } & $\begin{array}{c}\text { Before } \\
\text { intervention }\end{array}$ & $41.25 \pm 20.24$ & $43.94 \pm 22.56$ & 0.628 & 0.266 \\
\hline & $\begin{array}{c}\text { After } \\
\text { intervention }\end{array}$ & $34.16 \pm 18.67$ & $41.45 \pm 21.45$ & 1.813 & 0.036 \\
\hline
\end{tabular}

Note: $\mathrm{P}<0.05$ was statistically significant. 


\section{Discussion}

Breast cancer is a strong psychological stress for the patient itself. Breast cancer is a physical and mental disease. Due to psychosocial factors, most breast cancer patients have a series of psychological stress reactions such as anxiety, depression, fear and despair. These psychological stress responses not only affect the prognosis of breast cancer patients but also have a great impact on the patient's nervous system, endocrine system and immune system [4] [5]. Scholars have found that psychological stress may be mainly through changing the function of the hypothalamic-pituitary-adrenalin (HPA) axis and the sympathetic nervous system of the body. The HPA axis is hyperactive, which affects the activity of various immune cells, including NK cells, T lymphocytes, and macrophages [6] [7] [8]. When the immune cells of the body appear to have a balance disorder, cytokines can not only mediate chronic inflammation, but also aggravate the original psychological stress response, such as depression, anxiety, sleep disorders, fatigue or cognitive impairment, affecting the prognosis of the disease and reducing the quality of life [9] [10]. At present, the immune cytokines involved in the treatment of breast cancer at home and abroad mainly include interleukin, interferon and tumor necrosis factor, namely IL-2, IL-4, IL-6, IL-8, TNF- $\alpha$, etc, some cytokines. These cytokines are involved in stress regulation [11] [12] [13] [14]. In recent years, psychological intervention methods have been widely used in the treatment of cancer patients. Research [15] [16] [17] found that through psychological intervention in cancer patients, it could effectively alleviate the patient's psychological state, improve their immune function, and even improve the patient's life cycle.

Cognitive-behavioral intervention therapy is a group of short-term psychological interventions that alter the patient's poor cognition by changing the patient's thinking and behavior, and eliminate the patient's bad mood and cognition [18]. In recent years, it has been widely used in the treatment of breast cancer. Lan Yanhong [19] and other 82 patients with breast cancer chemotherapy were randomly divided into the control group and the observation group. The control group received the conventional chemotherapy program. The observation group implemented cognitive behavioral intervention therapy on this basis to correct the mistaken cognition of the patient. And to re-establish the patient's correct cognition, the results of cognitive behavioral intervention therapy in the observation group were significantly lower than the control group. Zeng Xiaomin [20] and other studies found that cognitive behavioral intervention therapy could effectively alleviate the depression and anxiety state of patients with chemotherapy after breast cancer surgery, and improve the immune function of patients. Xie Jun [21] and other cognitive behavior interventions in perioperative breast cancer patients, the results showed that the method could effectively improve the patient's psychological stress and improve the patient's immune function. The results of this study show that after cognitive behavioral intervention in breast cancer patients, their immune indicators have improved in different 
situations. The serum levels of IL-2, IL-4 and IL-8 in the intervention group were significantly lower than those before the intervention. The concentration of TNF- $\alpha$ was significantly higher than that before the intervention, and the serum IL-6 concentration did not change much. The reason was analyzed. The serum content of breast cancer patients may not only involve psychological factors, but also involve other factors of its own, such as the growth of the tumor itself; In addition, it may be related to the sample size and intervention time of this study. The serum levels of IL-2, IL-4 and IL-8 in the control group were lower than those in the serum before the intervention, but the decrease was not obvious; the IL- 6 concentration was higher than that before the intervention; The concentration of TNF- $\alpha$ increased compared with that before the intervention, but the change was not significant. This suggests that cognitive behavioral intervention therapy can improve the psychological stress response of breast cancer patients, promote the recovery of immune function, and promote the prognosis of the disease. TNF- $\alpha$ is mainly produced by macrophages and monocytes. It is a pleiotropic cytokine that plays an important role in maintaining immune homeostasis and promoting disease progression. It is a host defense pathogen and immune surveillance for malignant tumors. The cytokines necessary for cell proliferation are also important inflammatory mediators of various diseases. Up-regulation of TNF- $\alpha$ expression suggests that serum TNF- $\alpha$ levels are associated with the status and prognosis of breast cancer patients.

\section{Conclusion}

In summary, cognitive behavioral intervention therapy can effectively improve the immune function of breast cancer patients and has an important impact on the prognosis and outcome of patients, which is worthy of clinical promotion and application.

\section{Fund-Funded Projects}

2017 Hainan Natural Science Foundation No.: 817405.

\section{Conflicts of Interest}

The authors declare no conflicts of interest regarding the publication of this paper.

\section{References}

[1] Bray, F., Ferlay, J., Soerjomataram, I., et al. (2018) Global Cancer Statistics 2018: GLOBOCAN Estimates of Incidence and Mortality Worldwide for 36 Cancers in 185 Countries. CA: A Cancer Journal for Clinicians, 68, 394-424. https://doi.org/10.3322/caac.21492

[2] Chen, A.G., Li, W.J. and Yan, J. (2005) Effects of Psychosocial Factors Stress on Immune Function. Chinese Journal of Clinical Rehabilitation, 16, 134-135.

[3] Zhao, L., Wu, H., Zhou, X., Wang, Q., et al. (2012) Effects of Progressive Muscular Relaxation Training on Anxiety, Depression and Quality of Life of Endometriosis 
Patients under Gonadotrophin-Releasing Hormone Agonist Therapy. European Journal of Obstetrics \& Gynecology and Reproductive Biology, 162, 211-215. https://doi.org/10.1016/j.ejogrb.2012.02.029

[4] Zhang, H.M. and Zhang, J.P. (2010) The Influence of Psychosocial Factors on Immune Function and Its Psychological Intervention in Breast Cancer Patients. Nursing Research, 24, 2645-2647.

[5] Yang, J.J. and Liu, J.Q. (2014) Research Progress of Psychological Intervention on Immune Function of Breast Cancer Patients. Chinese Journal of Nursing, 49, 1243-1248.

[6] Lutgendorf, S.K., Sood, A.K., Antoni, M.H., et al. (2010) Host Factors and Cancer Progression: Biobehavioral Signaling Pathways and Interventions. Journal of Clinical Oncology, 28, 4094-4099. https://doi.org/10.1200/JCO.2009.26.9357

[7] Sexton, J.M., Scott, E.J., Deley, A.J., et al. (2014) Effects of an Exercise and Hypocaloric Healthy Eating Intervention on Indices of Psychological Health Status, Hypothalamic-Pituitary-Adrenal Axis Regulation and Immune Function after Early-Stage Breast Cancer: A Randomised Controlled Trial. Breast Cancer Research, 16, R39. https://doi.org/10.1186/bcr3643

[8] An, L.-Y., Tang, F. and Wang J.-X. (2015) Research Status of Relationship between Psychological Stress and Immune Function. Medical Review, 21, 414-416.

[9] Ryan, J.L., Carroll, J.K., Ryan, E.P., et al. (2007) Mechanisms of Cancer-Related Fatigue. The Oncologist, 12, 22-34. https://doi.org/10.1634/theoncologist.12-S1-22

[10] Janelsins, M.C., Mustian, K.M., Palesh, O.G., et al. (2012) Differential Expression of Cytokines in Breast Cancer Patients Receiving Different Chemotherapies: Implications for Cognitive Impairment Research. Supportive Care in Cancer, 20, 831-839. https://doi.org/10.1007/s00520-011-1158-0

[11] Jehn, C.F., Flath, B., Strux, A., et al. (2012) Influence of Age, Performance Status, Cancer Activity, and IL-6 on Anxiety and Depression in Patients with Metastatic Breast Cancer. Breast Cancer Research and Treatment, 136, 789-794. https://doi.org/10.1007/s10549-012-2311-2

[12] Nan, H. and Chang, Y.L. (2013) Research Progress of Psychological Intervention on Immune Function of Breast Cancer Patients. Chinese Journal of General Practice, 11, 1441-1442.

[13] Figueiredo-Braga, M., Cornaby, C., et al. (2018) Depression and Anxiety in Systemic Lupus Erythematosus: The Crosstalk between Immunological, Clinical, and Psychosocial Factors. Medicine, 97, e11376.

[14] Gold, M., Dunn, L.B., Phoenix, B., et al. (2016) Co-Occurrence of Anxiety and Depressive Symptoms Following Breast Cancer Surgery and Its Impact on Quality of Life. European Journal of Oncology Nursing, 20, 97-105. https://doi.org/10.1016/j.ejon.2015.06.003

[15] Han, R.Y., Wang, A.J., Zhang, D.J., et al. (2013) Effects of Cognitive Intervention on Perioperative Mood and Immune Function in Patients with Cervical Cancer. Journal of PLA University of Medical Sciences, 34, 371-372

[16] Peng, L., Xie, J.Z., Hu, Y., et al. (2015) Effects of Cognitive Behavioral Psychotherapy on Emotional and Immune Status in Patients with Lung Cancer. Modern Oncology Medicine, 23, 2953-2956.

[17] Li, J.J. and Hu, S.L. (2017) Effects of Psychological Behavior Intervention on Immune Function and Quality of Life in Patients with Lung Cancer. Hainan Medical Journal, 28, 2223-2225. 
[18] Liu, Z.D. (2011) Effects of Cognitive Behavioral Therapy on Quality of Life in Elderly Patients with Chronic Pain. Chinese Journal of Behavioral Medicine and Brain Science, 20, 830-832.

[19] Lan, Y.H., Deng, M.H. and Deng, F. (2012) Effects of Cognitive Behavioral Therapy on Quality of Life in Patients with Breast Cancer Chemotherapy. Chinese Journal of General Practice, 10, 1850-1851.

[20] Zeng, X.M. and Yang, W.B. (2018) Effects of Cognitive Behavioral Therapy on Psychological Status and Cellular Immune Function in Postoperative Chemotherapy Patients with Breast Cancer. Chinese Modern Doctor, 56, 94-97.

[21] Xie, J. and Shi, X.L. (2018) Effects of cognitive behavioral nursing on psychological, Cor and IL-2 levels in patients with breast cancer during perioperative period. Modern Journal of Integrated Traditional Chinese and Western Medicine, 27, 3976-3978+3990. 